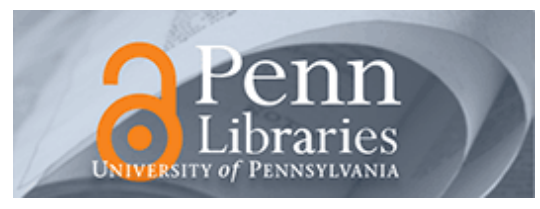

University of Pennsylvania ScholarlyCommons

8-2-2012

\title{
Optical Frequency Mixing Through Nanoantenna Enhanced Difference Frequency Generation: Metatronic Mixer
}

Uday K. Chettiar

University of Pennsylvania

Nader Engheta

University of Pennsylvania, engheta@ee.upenn.edu

Follow this and additional works at: https://repository.upenn.edu/ese_papers

Part of the Electrical and Computer Engineering Commons

\section{Recommended Citation}

Uday K. Chettiar and Nader Engheta, "Optical Frequency Mixing Through Nanoantenna Enhanced Difference Frequency Generation: Metatronic Mixer", . August 2012.

Chettiar, U. K. and Engheta, N. (2012). Optical frequency mixing through nanoantenna enhanced difference frequency generation: Metatronic mixer. Physical Review B, 86(7), 075405. doi: 10.1103/PhysRevB.86.075405 (C)2012 American Physical Society

This paper is posted at ScholarlyCommons. https://repository.upenn.edu/ese_papers/624

For more information, please contact repository@pobox.upenn.edu. 


\title{
Optical Frequency Mixing Through Nanoantenna Enhanced Difference Frequency Generation: Metatronic Mixer
}

\author{
Abstract \\ A design for a subwavelength all-optical frequency mixer is proposed. The method relies on enhanced \\ difference-frequency generation, which is achieved in two steps with the help of plasmonic \\ nanoantennas. The interaction of the two input signals with the nonlinear material is increased through \\ the use of input nanoantennas, which focus the incident energy of two different frequencies onto the \\ nanoparticle formed by a nonlinear material. Next, the difference-frequency emission is enhanced through \\ the Purcell effect by the use of a separate output nanoantenna that is resonant at the difference \\ frequency. The application of this twofold approach allows for a significant enhancement in the \\ difference-frequency generation efficiency. Simulation results are presented highlighting the features of \\ the method. This multi-element nanostructure is indeed an optical mixer circuit element in the metatronic \\ paradigm.

\section{Disciplines} \\ Electrical and Computer Engineering

\section{Comments} \\ Chettiar, U. K. and Engheta, N. (2012). Optical frequency mixing through nanoantenna enhanced \\ difference frequency generation: Metatronic mixer. Physical Review B, 86(7), 075405. doi: 10.1103/ \\ PhysRevB.86.075405 \\ (C2012 American Physical Society
}




\title{
Optical frequency mixing through nanoantenna enhanced difference frequency generation: Metatronic mixer
}

\author{
Uday K. Chettiar and Nader Engheta* \\ University of Pennsylvania Department of Electrical and Systems Engineering Philadelphia, Pennsylvania 19104 USA
}

(Received 6 March 2012; revised manuscript received 2 July 2012; published 2 August 2012)

\begin{abstract}
A design for a subwavelength all-optical frequency mixer is proposed. The method relies on enhanced difference-frequency generation, which is achieved in two steps with the help of plasmonic nanoantennas. The interaction of the two input signals with the nonlinear material is increased through the use of input nanoantennas, which focus the incident energy of two different frequencies onto the nanoparticle formed by a nonlinear material. Next, the difference-frequency emission is enhanced through the Purcell effect by the use of a separate output nanoantenna that is resonant at the difference frequency. The application of this twofold approach allows for a significant enhancement in the difference-frequency generation efficiency. Simulation results are presented highlighting the features of the method. This multi-element nanostructure is indeed an optical mixer circuit element in the metatronic paradigm.
\end{abstract}

DOI: 10.1103/PhysRevB.86.075405 PACS number(s): 61.46.-w, 78.67.Bf, 78.20.-e, 07.50.Ek

\section{INTRODUCTION}

A frequency mixer is a basic component in communications systems in which two input signals are multiplied, resulting in an output signal with the difference or sum of the frequencies of the two input signals. This circuit element is used extensively to modulate and demodulate signals in electronics. ${ }^{1,2}$ In optical communications, the mixing and modulation is usually accomplished through the use of electro-optical components. For example, optical heterodyne detection uses frequency mixing, but the output frequency is usually in the electronic range. ${ }^{3}$ The mixing is typically achieved by sending the two optical signals to a nonlinear photodetector, and the frequency mixed signal is available as the electrical signal across the photodetector, which is further extracted by applying a suitable filter. Here, we present an idea for an all-optical frequency subwavelength mixer which does not rely on electrical components. Instead, the frequency mixing is achieved through the use of optical second-order nonlinear material, while the response is enhanced by using plasmonic nanoantennas. Using numerical simulations, we demonstrate the possibility of generation of difference-frequency signal in this nanoscale optical mixer (although it should be noted that we can also easily modify this design to obtain the sum-frequency signal by appropriately tuning one of the nanoantennas to be resonant at the sum frequency).

In recent years, there has also been an increased interest in the nonlinear response of metamaterials, including second-harmonic generation (SHG), third-harmonic generation (THG), and Raman scattering (see e.g. Refs. 4-9). The enhanced nonlinear responses in subwavelength coated particles have also been studied for several years using quasistatic methods. ${ }^{10,11}$ It is well known that the secondorder susceptibility in optical nonlinear media is usually a very small parameter as compared with the first-order linear susceptibility. ${ }^{12}$ In order to observe a second-harmonic signal, therefore, one would need to have strong pump intensity. There have been various techniques for enhancing the second-order nonlinearity over the past few decades. With the advent of plasmonics, enhanced nonlinear processes have been observed in nanoplasmonic structures, including frequency mixing, ${ }^{13,14}$ harmonic generation, ${ }^{15-18}$ and electrically controlled nonlinear generation. ${ }^{19}$ Some of these results used a nonlinear material in conjunction with the plasmonic material, while others relied on the nonlinearity of the plasmonic material and nonlocal dielectric effects.

Most of these techniques rely on providing a localized region with enhanced field intensity termed as a hotspot. The hotspot effectively increases the pump field strength that is experienced by the nonlinear material. Due to the resonant coupling of optical signals with collections of conduction electrons at the surface of metallic nanostructures, the local field intensity can be enhanced in the vicinity of metallic nanostructures to create such hotspots. A simple and effective means of achieving this form of enhancement is to use a random metal-dielectric composite whose filling fraction is close to the percolation threshold. ${ }^{20}$ However, there is one more form of enhancement that can be exploited to further boost the second-order nonlinear response, namely the Purcell effect. $^{21}$ The Purcell effect states that the rate of spontaneous emission could be modified by the environment in terms of photon density of states. For example, if the environment of an emitter does not support any mode at the emission wavelength, it will result in a complete suppression of emission. On the other hand, if the environment supports an increased number of modes compared to free space modes, it will lead to an enhancement of emission. Strictly speaking, the Purcell effect deals with the change in spontaneous emission rate, but it has been shown that the rate of change in spontaneous emission rate is equal to the rate of change in the energy emission by a dipole. ${ }^{22}$ Since the nonlinear emission can be modeled by a polarization density term, it implies that the Purcell effect will also change the nonlinear emission in proportion to the change in spontaneous emission rate. While using hotspots to enhance the difference-frequency (DF) signal, the resulting induced nonlinear dipoles may radiate into a region where nanostructures may not interact with the difference-frequency generation (DFG) frequency, and consequently, we would fail to achieve any additional enhancement due to the Purcell effect. In this paper, we explore the possibility of designing a nanostructure in the form of three (or two) nanoantennas 


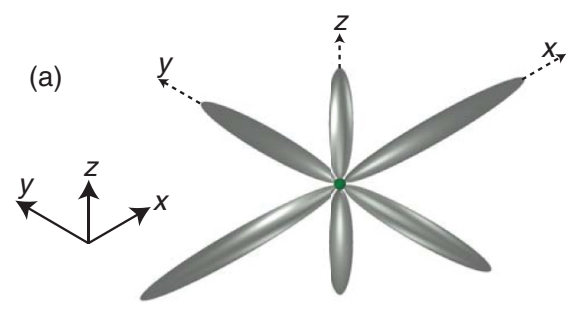

(b)

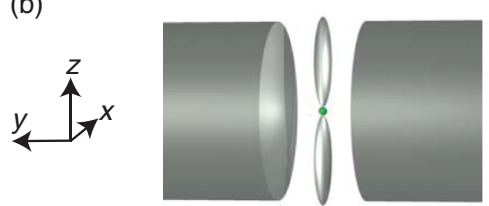

FIG. 1. (Color online) Schematic of the subwavelength optical difference-frequency-generation (DFG) mixers using proper combinations of nanoantennas. (a) When the frequencies of the two input signals differ significantly, we do need two input and one output nanoantenna. (b) However, when the two input frequencies are very close to each other, we may have only one input and one output nanoantenna.

designed to be at resonance at two input frequencies and at the output difference frequency, respectively. The plasmonic nanoantennas can be either cylindrical or ellipsoidal in shape. Such nanoantennas have been studied in great detail by various groups. ${ }^{23-25}$ Plasmonics nanoantennas have also been shown theoretically ${ }^{26}$ and experimentally ${ }^{27}$ to enhance spontaneous emission rate in their vicinity. This shows the possibility that nanoantennas could also be used to enhance nonlinear processes through the Purcell effect. The two input nanoantennas would focus the two pump signals with two different frequencies to a hotspot where the nonlinear material will be located, and the output (third) nanoantenna would interact with the nonlinear material in order to enhance the emission at the DFG frequency through the Purcell effect. When the two input frequencies are very close to each other, one could use a single nanoantenna as the input nanoantenna for both input frequencies, while the output antenna would still provide the enhancement of the DF signal through the Purcell effect. This nanoantenna-enhanced optical mixer may be considered as a mixing circuit element in optical metatronic circuitry, i.e. metamaterial-inspired optical nanocircuits. ${ }^{28-31}$

The device is schematically illustrated in Fig. 1, where the top schematic shows the structure with three nanoantennas. When the two input frequencies $\left(\omega_{1}\right.$ and $\left.\omega_{2}\right)$ are well separated, we would need two separate input antennas for enhancement, and thus two of the three antennas in the top panels are designed for enhancement of the signals with frequencies $\omega_{1}$ and $\omega_{2}$. The third nanoantenna is designed for enhancement of the difference-frequency signal. The bottom schematic indicates the situation where the two input frequencies are very close to each other, and hence they can share the same input nanoantenna. In this case, the difference frequency $\left(\omega_{1}-\omega_{2}\right)$ is much lower than either input frequency. Hence, the output antenna is much longer than the input antenna. Because of the orientation of the antennas in this mixer, the second-order nonlinear material needs to have specific symmetries in order for the input and output polarizations to align with the nanoantennas. For example, in the first case [Fig. 1(a)], the component of the susceptibility tensor of interest is given by $\chi_{i j k}^{(2)}\left(\omega_{1}-\omega_{2} ; \omega_{1}, \omega_{2}\right)$, where $i \neq j \neq k$, whereas in the second case (bottom panel), the component of interest is given by $\chi_{i j j}^{(2)}\left(\omega_{1}-\omega_{2} ; \omega_{1}, \omega_{2}\right)$, where $i \neq j$. The DF polarization term is given by $P\left(\omega_{1}-\omega_{2}\right)=2 \varepsilon_{o} \chi^{(2)} \boldsymbol{E}_{\omega_{1}} \boldsymbol{E}_{\omega_{2}}^{*} \cdot{ }^{12}$ One can easily note that the DF polarization term is proportional to the product of the two input signals. This can be used to modulate or demodulate a signal on a carrier.

\section{RESONANCE OF SPHEROIDS}

In order to gain a qualitative idea of the resonance characteristics of spheroids, we will present a brief study on these resonant modes using simulations and theory based on quasistatic approximation. The polarizability for an ellipsoid under quasistatic approximation can be described using the depolarization factor given by the following expression: ${ }^{32}$

$$
L_{1}=\int_{0}^{\infty} \frac{a b c}{2\left(s+a^{2}\right)^{3 / 2}\left(s+b^{2}\right)^{1 / 2}\left(s+c^{2}\right)^{1 / 2}} d s .
$$

In the above equation, $a, b$, and $c$ represents the semiaxes lengths of the ellipsoid, and $L_{1}$ represents the depolarization factor along axis $a$. Similar expressions could be written for the depolarization factors along other directions. In the case of a prolate spheroid $(a>b=c)$, the depolarization factor can be written in the following form:

$$
L_{1}=\frac{1-e^{2}}{e^{2}}\left[-1+\tanh ^{-1}(e)\right],
$$

where $e^{2}=1-(b / a)^{2}{ }^{32}$ The polarizability of the ellipsoid $(\alpha=p / E$ ) can be expressed in the following form:

$$
\alpha_{1}=4 \pi \varepsilon_{m} a b c \frac{\varepsilon_{1}-\varepsilon_{m}}{3 \varepsilon_{m}+3 L_{1}\left(\varepsilon_{1}-\varepsilon_{m}\right)},
$$

where $\varepsilon_{1}$ and $\varepsilon_{m}$ are the permittivity of the ellipsoid and the surrounding medium, respectively. The ellipsoid undergoes resonance when the real part of the denominator goes to zero. Assuming the surrounding medium to be lossless, this results in the following condition for resonance:

$$
\operatorname{Re}\left(\varepsilon_{1}\right)=-\varepsilon_{m} \frac{1-L_{1}}{L_{1}} .
$$

To test this theory, we performed a series of simulations with spheroids of various aspect ratios. The full-wave numerical simulations were performed using a commercial finite-element-method- (FEM) based solver (COMSOL Multiphysics). The meshing was done with tetrahedral cubic elements. The simulation domain is surrounded by a perfectly matched layer (PML) in order to absorb the outgoing waves. The scattering cross sections are evaluated by integrating the intensity of the scattered wave over a closed surface enclosing the scatterer. The scattered power thus obtained is divided by the incident intensity to yield the scattering cross section. The relative permittivity of silver is calculated using the standard Drude model, i.e. $\varepsilon(\omega)=\varepsilon_{\infty}-\frac{\omega_{p}^{2}}{\omega(\omega+i \gamma)}$, with realistic 
losses obtained from experimental data in the literature. The parameter values are as follows: $\varepsilon_{\infty}=5.0, \omega_{p}=$ $9.2159 \mathrm{eV}\left(f_{p}=2.228 \times 10^{15} \mathrm{~Hz}\right)$, and $\gamma=0.0212 \mathrm{eV}$ $\left(f_{\gamma}=5.12 \times 10^{12} \mathrm{~Hz}\right){ }^{33}$

Figure 2 shows the results of simulations where the minor axis of the spheroid was fixed at $20 \mathrm{~nm}$, and the major axis (a) was varied between 20 and $100 \mathrm{~nm}$. Figure 2(a) shows the scattering efficiency of the spheroid as a function of the wavelength and the major axis of the spheroid. The scattering efficiency is defined as the ratio of the scattering cross section to the geometrical cross section. Figure 2(b) shows the resonance wavelength as a function of the major axis obtained using the simulation data and the analytical resonance wavelength obtained using Eq. (2). The simulation and analytical plots match reasonably well except for a small red shift of around $20 \mathrm{~nm}$ in the simulation results. Figure 2(c) shows the plots of the scattering efficiency as a function of the wavelength for the spheroids of various lengths. Figure 2(d) shows the resonance mode profile for a 100-nm-long spheroid at a wavelength of $660 \mathrm{~nm}$.

In order to describe the resonance of a pair of spheroids, we can use the coupled dipole approximation along with the polarizability calculated for the single spheroids. If two dipoles with polarizability given by $\alpha_{1}$ and $\alpha_{2}$ are located at $\mathbf{r}_{1}$ and $\mathbf{r}_{2}$, the effective dipole moment of the two polarizable dipoles can be written as follows:

$$
\begin{gathered}
\mathbf{p}_{1}=\alpha_{1}\left[\mathbf{E}_{0}+\overline{\overline{\mathbf{G}}}\left(\mathbf{r}_{1}, \mathbf{r}_{2}\right) \mathbf{p}_{2}\right] \\
\mathbf{p}_{2}=\alpha_{2}\left[\mathbf{E}_{0}+\overline{\overline{\mathbf{G}}}\left(\mathbf{r}_{2}, \mathbf{r}_{1}\right) \mathbf{p}_{1}\right],
\end{gathered}
$$

where $\overline{\overline{\mathbf{G}}}$ represents the dyadic Green's function and $\mathbf{E}_{0}$ represents the incident field. When both the spheroids are linearly aligned along with the polarization of the incident electric field, the above equation can be simplified by considering only the relevant component of the various vectors and the Green's function. Under this approximation, we can write the dipole moment as follows:

$$
p_{1}=\alpha_{1} \frac{\left[1+\alpha_{1} G\left(\mathbf{r}_{1}, \mathbf{r}_{2}\right)\right]}{\left[1-\alpha_{1} \alpha_{2} G\left(\mathbf{r}_{1}, \mathbf{r}_{2}\right) G\left(\mathbf{r}_{2}, \mathbf{r}_{1}\right)\right]} E_{0} .
$$

When both the spheroids are identical, this expression could be further simplified by setting $\alpha_{1}=\alpha_{2}$ and utilizing the reciprocity in Green's function:

$$
p=\frac{\alpha E_{0}}{1-\alpha G\left(\mathbf{r}_{1}, \mathbf{r}_{2}\right)} .
$$

This yields the following formula for the effective polarizability:

$$
\alpha_{e}=\frac{\alpha}{1-\alpha G}
$$
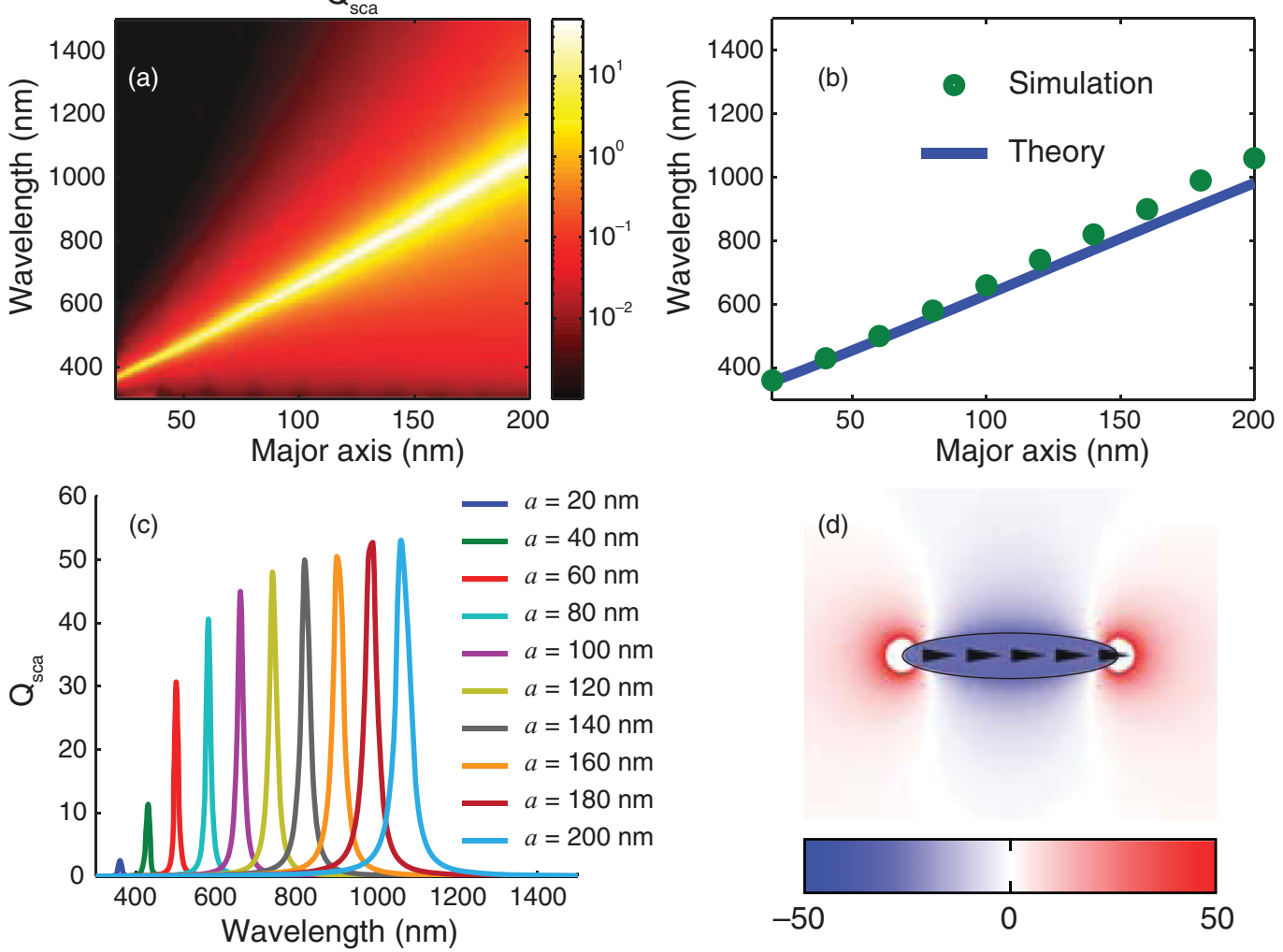

FIG. 2. (Color online) (a) Scattering efficiency (log scale) as a function of wavelength and the major axis (length) of the spheroid. (b) Resonance wavelength as a function of the major axis. (c) Scattering efficiency (linear scale) as a function of the wavelength for spheroids with various major axis, $a$. (d) Field map for a spheroid under resonance ( $a=100 \mathrm{~nm}, \lambda=660 \mathrm{~nm}$ ). The color represents the electric field component along the major axis, and the arrows represent the direction of the electric displacement vector. For all results, the minor axis of the prolate spheroid is fixed at $20 \mathrm{~nm}$. 
We have dropped the coordinate inputs in the Green's function for the sake of brevity. Using the formula for $\alpha$ from Eq. (1) and setting the denominator of $\alpha_{e}$ to zero yields the following resonance condition for the coupled spheroids:

$$
\operatorname{Re}\left(\varepsilon_{1}\right)=-\varepsilon_{m} \frac{\left(1-L_{1}+V G \varepsilon_{m}\right)}{\left(L_{1}-V G \varepsilon_{m}\right)},
$$

where $V$ is the volume of the spheroid. The above equation assumes the $G$ is real, which is a good approximation when the spheroids are located close to each other. In the general case, we could easily account for a complex $G$ by directly equating the real part of the denominator of $\alpha_{e}$ to zero. From Eq. (3), we can see that the coupling between the two spheroids, represented by $G$, serves to push the resonance to more negative values of $\varepsilon_{1}$. For a Drude type material, this implies a red shift in the resonance wavelength. To test this theory, we performed a series of simulations where two spheroids (major axis $=100 \mathrm{~nm}$, minor axis $=20 \mathrm{~nm}$ ) were separated by a gap size ranging from 5 to $100 \mathrm{~nm}$. The results are shown in Fig. 3. Figure 3(a) shows the scattering efficiency as a function of the wavelength and the gap size. As expected, we see that the resonance wavelength shifts towards longer wavelengths as the gap size is decreased. Figure 3(b) shows a comparison between the simulated resonance wavelength and the analytical equation. Two adjustments had to be performed on Eq. (3); first, a red shift of $20 \mathrm{~nm}$ was added to account for the slight mismatch between the simulated and analytical resonance wavelength for single spheroids, as evident from Fig. 2(b). Secondly, the coupling factor $(G)$ was reduced by a factor of 3. This was done to account for the fact that the spheroids have a finite extent, whereas Eq. (3) was derived by assuming that the spheroids behave like point dipoles. Hence, the coupling factor $G$ gets averaged over the finite extent of the spheroid, resulting in a lower value. Due to this, although the analytical expression reproduces the trend really well, it does not provide good quantitative agreement with the full-wave simulations, unless we use a fitting parameter to account for the averaging effect caused by the finite size of the spheroids. Reducing $G$ by a factor of 3 provided the best fit with the full-wave simulation results. Figure 3(c) plots the scattering efficiency as a function of the wavelength for selected gap sizes. We can see that, even though the resonance wavelength shifts with the gap size, the maximum scattering efficiency does not show a lot of variation. Figure 3(d) shows the resonance mode profile for a gap size of $20 \mathrm{~nm}$ at a wavelength of $683 \mathrm{~nm}$.

From the above results, we can infer that, although changing the gap size changes the resonance wavelength of the antenna, the shift is not very significant until very small gap sizes are reached. Hence, relying solely on the gap size to tune the resonance of a nanoantenna is not very practical. For this reason, we use both the length of the spheroid and the gap size to achieve resonance at the desired wavelength.
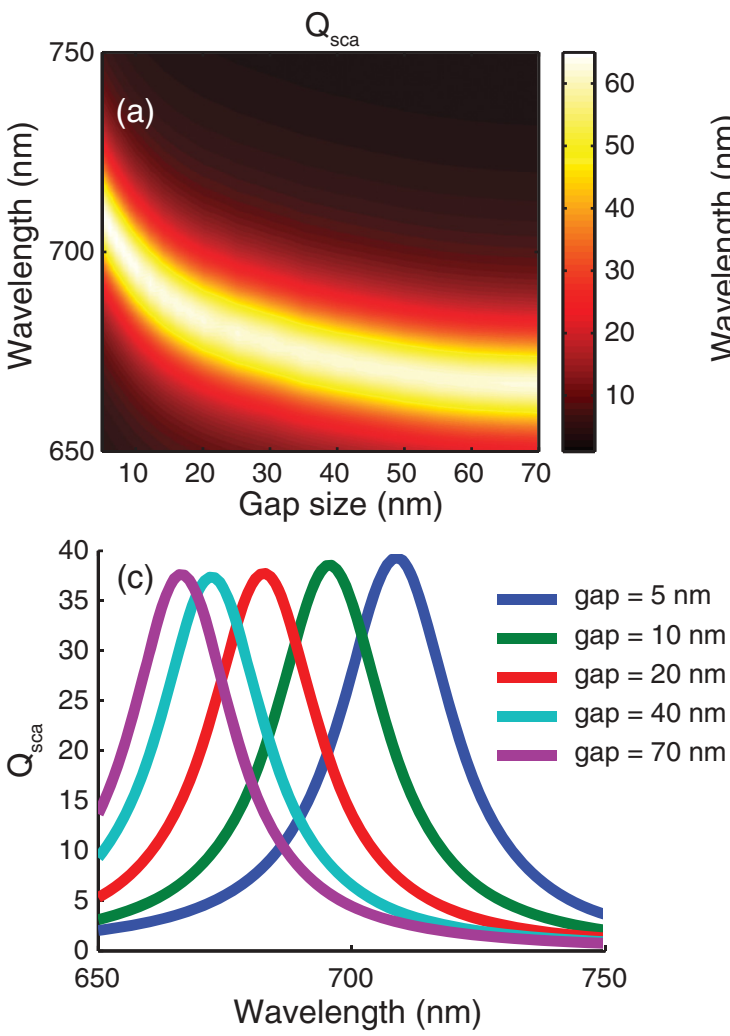

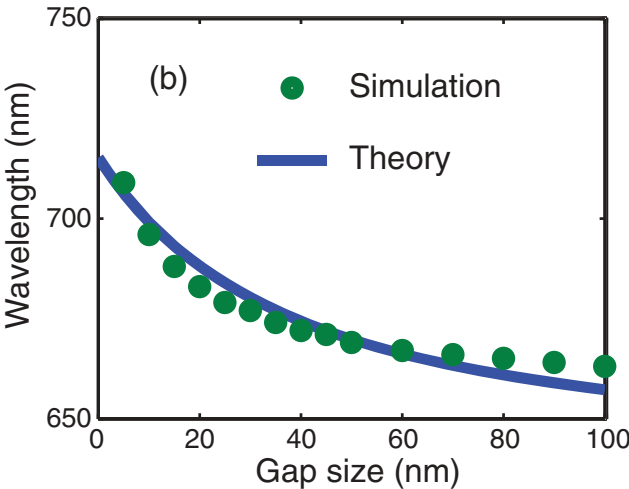

(d)

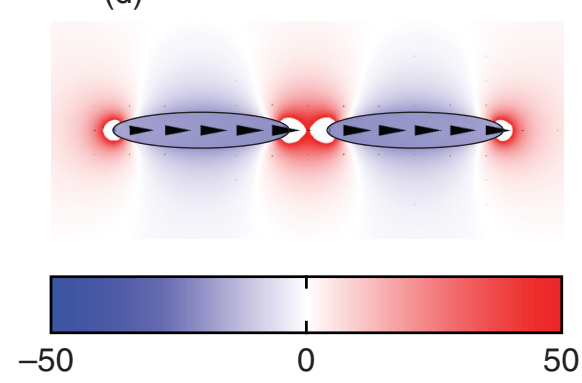

FIG. 3. (Color online) (a) Scattering efficiency (linear scale) as a function of wavelength and the gap size. (b) Resonance wavelength as a function of the gap size. (c) Scattering efficiency (linear scale) as a function of the wavelength for spheroid pairs with various gap size. (d) Field map for a spheroid under resonance (gap size $=20 \mathrm{~nm}, \lambda=683 \mathrm{~nm}$ ). The color represents the electric field component along the major axis, and the arrows represent the direction of electric displacement vector. For all results, the major and minor axes of the two prolate spheroids are fixed at 100 and $20 \mathrm{~nm}$, respectively. 


\section{RESULTS FOR THREE-ANTENNA STRUCTURE}

Having analyzed the individual spheroids and paired spheroid, we now move to the three-nanoantenna structure shown in Fig. 1(a). The three nanoantennas are assumed to be made of silver, and all the nanoantennas are designed in the shape of prolate spheroids. The input antenna for the higher frequency $\left(\omega_{1}\right)$ has a major axis of $100 \mathrm{~nm}$ and minor axis of $20 \mathrm{~nm}$. The input antenna for the lower frequency $\left(\omega_{2}\right)$ has a major axis of $200 \mathrm{~nm}$ and minor axis of $20 \mathrm{~nm}$. The output antenna, which is used to enhance the DF signal, is the longest with a major axis of $280 \mathrm{~nm}$ and minor axis of $20 \mathrm{~nm}$. The gap for all three antennas is set at $12 \mathrm{~nm}$. The nonlinear material is assumed to be a sphere of radius $5 \mathrm{~nm}$. Because of the symmetry requirements for $\chi^{(2)}$, the material is assumed to be GaAs, which belongs to crystal class $\overline{4} 3 m$ $\left(d_{16}=\frac{\chi_{x y z}^{(2)}}{2}=370 \mathrm{pm} / \mathrm{V}\right) .^{12}$

In order to arrive at the final dimensions of all the antennas, the initial optimization is carried out by calculating the scattering cross section of the triple-antenna structure, in the absence of the nonlinearity in the particle at the gap, for the three different incident polarizations corresponding to the two input frequencies and one output frequency. The scattering cross section (SCS) plots are shown in Fig. 4.

We see that the input antenna for the higher input frequency has two resonances (around $\lambda=700$ and $800 \mathrm{~nm}$ ), and the input antenna for the lower input frequency has one resonance (around $\lambda=1260 \mathrm{~nm}$ ). The third antenna, which is designed for the output DF signal, has a resonance at a wavelength of $1700 \mathrm{~nm}$, which is very close to the wavelength corresponding to the difference frequency $(1575 \mathrm{~nm})$ when the two inputs are set to 700 and $1260 \mathrm{~nm}$. The scattering cross section line shapes in Fig. 4 are markedly more complicated than the line shapes in Figs. 2 and 3. This happens due to the fact that, in this case, there are three antennas, and there is some degree of coupling between them. While deciding on which resonances are appropriate for an antenna application, it is also important to look at the electric field in the antenna gap where the nonlinear particle is placed. The electric field enhancement (inside the nonlinear particle at the gap but with the nonlinear susceptibility set to zero for now) corresponding to the scattering cross sections are shown in Fig. 5.

Next, we put back the nonlinear susceptibility in the particle at the gap, and we consider the full-wave nonlinear simulation in order to calculate the DFG conversion cross section. For this simulation, the incident field is set to $10^{6} \mathrm{~V} / \mathrm{m}$. In order to quantitatively study the effect of the three antennas, we also consider a couple of control cases. In the first case, we assume we only have the input antenna for higher frequency, but no input antenna for the lower input frequency and no output antenna. In the second case, we assume we have the two input antennas but no output antenna. Finally, in the third case, we assume all three antennas. The results are shown in Fig. 6.
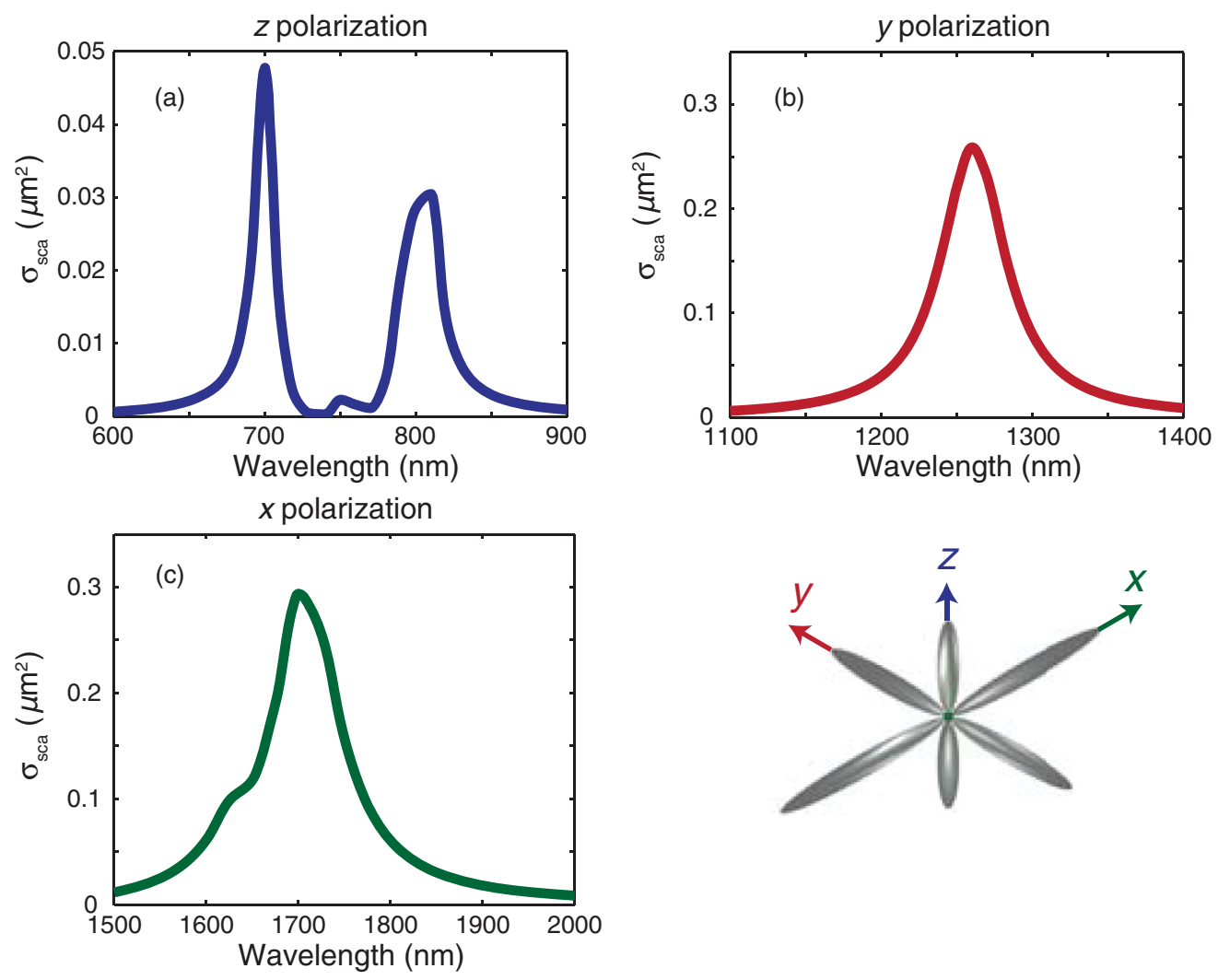

FIG. 4. (Color online) Scattering cross sections of the three-antenna structure shown in Fig. 1(a), for the cases of (a) $z$-oriented polarization for the incident signal with radian frequency $\omega_{1}$, (b) $y$-oriented polarization for the incident signal with radian frequency $\omega_{2}$, and (c) $x$-oriented polarization for the incident signal with radian frequency $\omega_{1}-\omega_{2}$. For the evaluation of the scattering cross section, the nonlinearity of the material in the particle is assumed to be zero. The schematic shows the coordinate directions with the three antennas. 

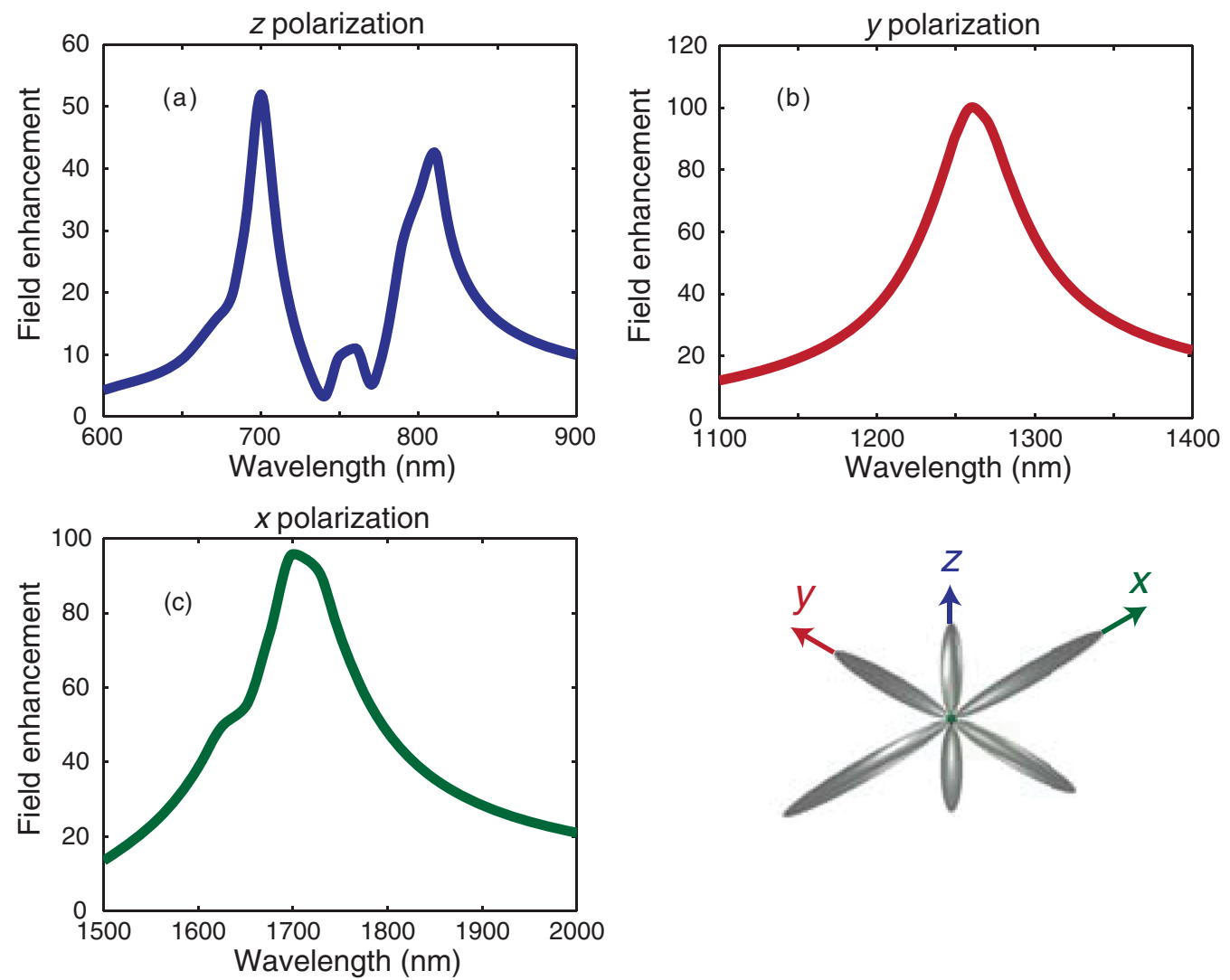

FIG. 5. (Color online) Field enhancement inside the nonlinear particle, i.e. the ratio of the magnitude of the electric field inside the nanoparticle and the magnitude of the incident electric field, for the cases shown in Fig. 2, i.e. (a) $z$-oriented polarization for the incident signal with radian frequency $\omega_{1}$, (b) $y$-oriented polarization for the incident signal with radian frequency $\omega_{2}$, and (c) $x$-oriented polarization for the incident signal with radian frequency $\omega_{1}-\omega_{2}$. For the evaluation of the field enhancement, the nonlinearity of the material in the particle is assumed to be zero. The schematic shows the coordinate directions with the three antennas.

In this figure, the DFG cross sections are plotted as a function of two input frequencies $\left(v_{1}\right.$ and $v_{2}$ ) shown on two axes. The DFG cross section is defined as the ratio of the total DF power and the geometric mean of the intensities for the two inputs. With this representation, the enhancement due to the Purcell effect would show up as a straight line. For the first control case (with only $\omega_{1}$ antenna present), we can only see the lightning rod effect due to the input antenna corresponding to $\omega_{1}$. Note that we see two peaks corresponding to the two resonances of the $\omega_{1}$ antenna, as also shown in Fig. 4, for the $z$ polarization. For the second control case, we see the lightning rod effect from both input rods, and we see a stronger enhancement in the DFG signal when the lighting rod effects from the two input antennas overlap. There is a four-orders-of-magnitude enhancement in the DFG cross section compared to the first control case. Finally, when we have all three antennas present, we can see the Purcell effect enhancement from the output antenna in addition to the two lighting rod effects from the two input antennas. The Purcell effect manifests itself in the form of diagonal lines corresponding to the straight lines given by $v_{1}-v_{2}=v_{3 \text {,res }}$, where $v_{3 \text {,res }}$ is the resonance frequency of the output antenna. We also see another four-orders-of-magnitude enhancement in the final case when compared to the second control case.

The field enhancement due to the first antenna is around 50, suggesting an enhancement of 2500 in the DFG emission due to the first antenna. The second input antenna provides the field enhancement of about 100, resulting in an increase of factor 10,000 in the DFG emission, and this is the four orders of magnitude enhancement we note due to the second antenna in Fig. 6(b). The third (i.e. output) antenna gives a field enhancement of another 100, thus another four-ordersof-magnitude enhancement in the emission. Overall, the total enhancement in the DFG signal from all three antennas is around $2.5 \times 10^{11}$.

\section{RESULTS FOR TWO-ANTENNA STRUCTURE}

When the two input signals have frequencies that are near each other in the spectral domain, the first two antennas can be replaced with one single antenna for the field enhancement of both input signals. However, in this case, the difference frequency can be relatively low, e.g. in the $\mathrm{THz}$ regime, and thus the output antenna for the Purcell enhancement may be rather large. Figure 1(b) shows the sketch of this scenario. The antennas are set to silver with the same permittivity as in the previous sections. The nanoantenna (input antenna) is designed in the shape of a prolate spheroid with a major axis of $100 \mathrm{~nm}$ and a minor axis of $20 \mathrm{~nm}$. The output antenna, on the other hand, is shaped in the form of a cylinder with a radius of $100 \mathrm{~nm}$. Each arm of the output antenna is $7.5 \mu \mathrm{m}$ in length. The length is chosen to be a quarter of the output wavelength 

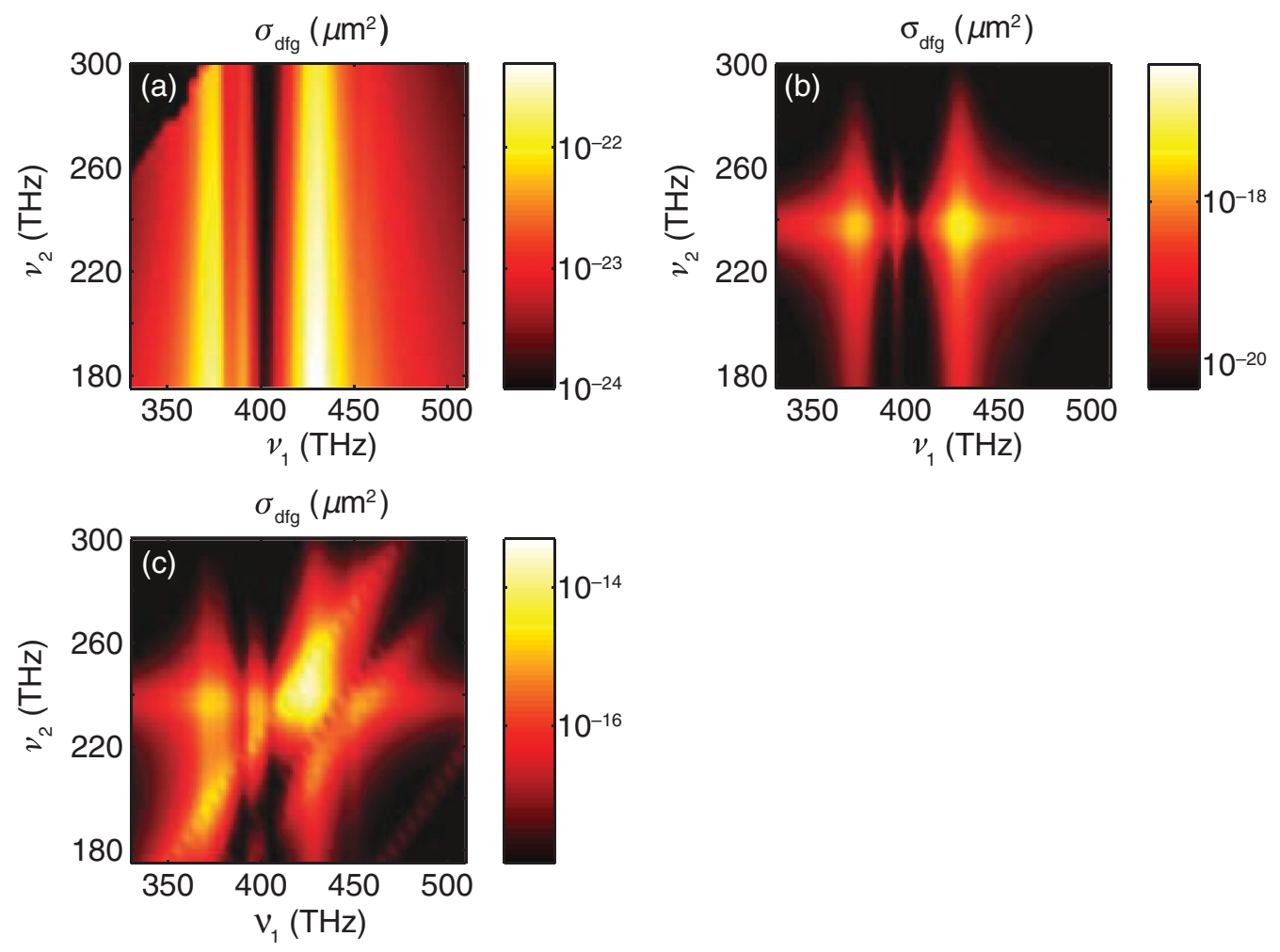

FIG. 6. (Color online) DFG emission cross sections for the three-antenna structure with the nonlinear particle in the gap (a) with only $\omega_{1}$ antenna present, (b) with $\omega_{1}$ and $\omega_{2}$ antennas present, and (c) with all three antennas present. These simulations include nonlinearity.

$(\lambda=30 \mu \mathrm{m})$. The gap sizes for the input and output antennas are set at 12 and $200 \mathrm{~nm}$, respectively. The nonlinear material is a sphere of radius $5 \mathrm{~nm}$. In this geometry, both the input signals use the same nanoantenna in order to achieve enhancement via the lightening rod effect. The output signal is enhanced via Purcell effect using the output antenna. The symmetry requires the $\chi^{(2)}$ of the material to be of form $\chi_{i j j}^{(2)}$, where $i \neq j$. Such symmetry is followed by materials belonging to the crystal class $m m 2, m, 3,3 m$, etc. For the simulations in this work, we assume the material to be potassium titanyl phosphate (KTP), which belongs to crystal class $m m 2\left(d_{23}=\right.$ $\left.\frac{\chi_{z y y}^{(2)}}{2}=6.5 \mathrm{pm} / \mathrm{V}\right) .^{34}$

The dimensions of the antennas are optimized by monitoring the scattering cross sections and the field enhancements inside the nonlinear material for incident radiation at input frequencies. In this case, we have one control case where the output antenna is removed from the system. Since the scattering cross section is dominated by the output antenna when it is present, here we show the scattering cross section only for the control case in Fig. 7(a). However, for the field enhancement inside the nonlinear particle, we can compare the two scenarios, as presented in Fig. 7(b).

We see a strong resonance at both 700 and $800 \mathrm{~nm}$. The scattering cross section in Fig. 7(a) looks somewhat different than that in Fig. 4(a), even though the $z$-oriented antenna in the three-antenna structure is identical to the $z$-oriented antenna in the two-antenna structure. This difference is caused by the fact that, in Fig. 4(a), all three antennas were taken into account, whereas in Fig. 7(a), we ignored the $\mathrm{THz}$ output antenna in order to see more clearly the scattering response of the input antenna. The field enhancement inside the nonlinear material seems to be more robust at $800 \mathrm{~nm}$ and is around 30 even in the presence of the large output antenna. If we assume that the output frequency is set to 10 $\mathrm{THz}(\approx 30 \mu \mathrm{m}$ wavelength) and the higher frequency input signal at a wavelength of $800 \mathrm{~nm}$, the lower frequency input signal should be at a wavelength of around $822 \mathrm{~nm}$ since the difference-frequency signal corresponding to input signals at 800 and $822 \mathrm{~nm}$ has a wavelength of $30 \mu \mathrm{m}$. Hence, the resonance should have a bandwidth of at least $20 \mathrm{~nm}$ in order to simultaneously enhance both input signals. In this case, the bandwidth of the resonance is around $40 \mathrm{~nm}$.

Next, we utilize the full-wave simulation in order to calculate the DFG conversion efficiency. As in the previous case, here the incident field is set to $10^{6} \mathrm{~V} / \mathrm{m}$. The results are plotted in Fig. 7(c). We see an enhancement of 600 folds when the output antenna is present. This is in addition to the enhancement caused by the input antenna, which can be easily estimated by using field enhancement values from Fig. 7(b), and it comes out to be around a factor of $10^{5}$. Therefore, the overall enhancement of seven orders of magnitude may be achieved by using the two-antenna structure here.

\section{SUMMARY AND CONCLUSION}

Using a series of full-wave numerical simulations, we have demonstrated a nano- (or micro- in case of THz output) scale all-optical frequency mixer that provides the product of two optical signals at the difference frequency. The difference frequency could lie either in the optical regime or in the 

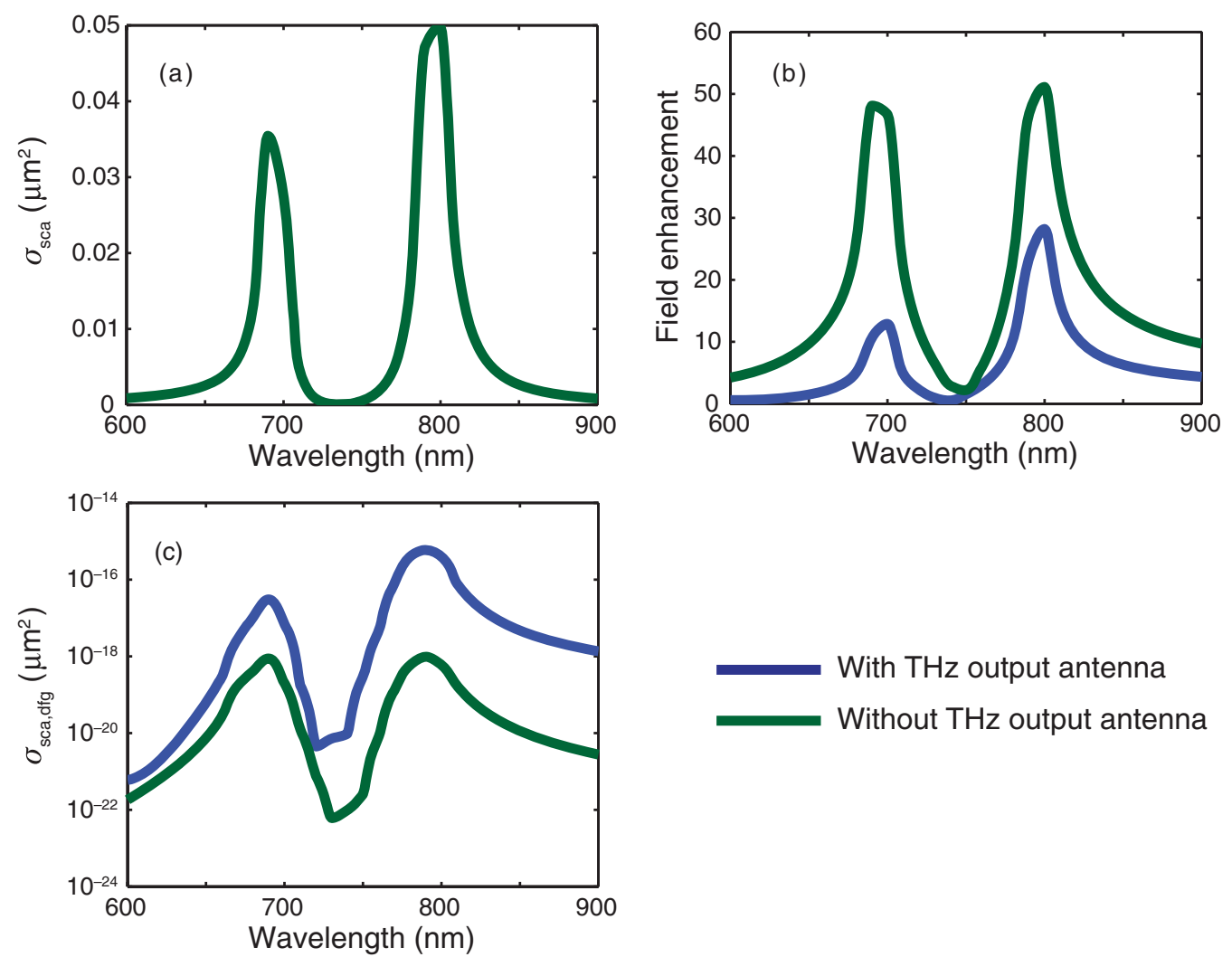

FIG. 7. (Color online) Scattering cross section, field enhancement, and DFG emission cross section for the two-antenna structure shown in Fig. 1(b): (a) Scattering cross section for the control structure, i.e. where the output antenna is absent. (b) Electric field enhancement inside the nonlinear material with and without the output antenna. (c) DFG emission cross section with and without the THz output antenna. The nonlinearity in the particle is set to zero for the evaluation of the scattering cross section and the field enhancement.

$\mathrm{THz}$ range. Overall, the output signal may be enhanced by a factor of around $10^{11}$ in the case where the output frequency is in the optical range, and by a factor of $10^{7}$ when the output frequency is in the $\mathrm{THz}$ range. By tuning the output antenna to be resonant at the sum frequency, we can easily convert the device to output the product at the sum frequency $\left(\omega_{1}+\omega_{2}\right)$ instead of the difference frequency $\left(\omega_{1}-\omega_{2}\right)$. The output signal could be further sent to optical amplifiers in order to form an all-optical communication system that could also entirely modulate and demodulate signals in the optical domain. Analogous to the mixer element in electronic circuits, the structure we investigated here may be considered as another subwavelength optical lumped circuit element in the optical metatronic paradigm. This structure may also be utilized as a building block in the design of bulk nonlinear optical metamaterials in which two optical signals may be mixed in order to generate different-frequency signals.

\section{ACKNOWLEDGMENTS}

This work is supported in part by the US Office of Naval Research (ONR) Multidisciplinary University Research Initiative (MURI) Grant No. N00014-10-1-0942.
*Corresponding author: engheta@ee.upenn.edu

${ }^{1}$ J. G. Proakis and M. Salehi, Communication Systems Engineering, 2nd ed. (Prentice Hall, Upper Saddle River, NJ, 2002).

${ }^{2} \mathrm{P}$. Horowitz and W. Hill, The Art of Electronics, 2nd ed. (Cambridge University Press, Cambridge, 1989).

${ }^{3}$ R. A. Linke and A. H. Gnauck, J. Lightwave Technol. 6, 1750 (1988).

${ }^{4}$ M. W. Klein, C. Enkrich, M. Wegener, and S. Linden, Science 313, 502 (2006).

${ }^{5}$ M. W. Klein, M. Wegener, N. Feth, and S. Linden, Opt. Express 15, 5238 (2007).
${ }^{6}$ A. K. Popov and V. M. Shalaev, Appl. Phys. B 84, 131 (2006).

${ }^{7}$ I. V. Shadrivov, A. B. Kozyrev, D. W. van der Weide, and Y. S. Kivshar, Opt. Express 16, 20266 (2008).

${ }^{8}$ C. Argyropoulos, P. Y. Chen, G. D’Aguanno, N. Engheta, and A. Alù, Phys. Rev. B 85, 045129 (2012).

${ }^{9}$ P. Y. Chen and A. Alù, Phys. Rev. B 82, 235405 (2010).

${ }^{10}$ N. Kalyaniwalla, J. W. Haus, R. Inguva, and M. H. Birnboim, Phys. Rev. A 42, 5613 (1990).

${ }^{11}$ J. W. Haus, N. Kalyaniwalla, R. Inguva, M. Bloemer, and C. M. Bowden, J. Opt. Soc. Am. B 6, 797 (1989). 
${ }^{12}$ R. Boyd, Nonlinear Optics, 3rd ed. (Academic Press, Boston, 2008).

${ }^{13}$ M. Danckwerts and L. Novotny, Phys. Rev. Lett. 98, 026104 (2007).

${ }^{14}$ P. Genevet, J. P. Tetienne, E. Gatzogiannis, R. Blanchard, M. A. Kats, M. O. Scully, and F. Capasso, Nano Lett. 10, 4880 (2010).

${ }^{15}$ K. Chen, C. Durak, J. R. Heflin, and H. D. Robinson, Nano Lett. 7, 254 (2007).

${ }^{16}$ W. Fan, S. Zhang, N. C. Panoiu, A. Abdenour, S. Krishna, R. M. Osgood, K. J. Malloy, and S. R. J. Brueck, Nano Lett. 6, 1027 (2006).

${ }^{17}$ S. Kim, J. Jin, Y. J. Kim, I. Y. Park, Y. Kim, and S. W. Kim, Nature 453, 757 (2008).

${ }^{18}$ K. Thyagarajan, S. Rivier, A. Lovera, and O. J. F. Martin, Opt. Express 20, 12860 (2012).

${ }^{19}$ W. Cai, A. P. Vasudev, and M. L. Brongersma, Science 333, 1720 (2011).

${ }^{20}$ V. M. Shalaev, Nonlinear Optics of Random Media: Fractal Composites and Metal-dielectric Films (Springer, Berlin; New York, 2000).

${ }^{21}$ E. M. Purcell, Phys. Rev. 69, 681 (1946).
${ }^{22}$ L. Novotny and B. Hecht, Principles of Nano-optics (Cambridge University Press, Cambridge, 2006).

${ }^{23}$ A. Alù and N. Engheta, Nat. Photonics 2, 307 (2008).

${ }^{24}$ M. Schnell, A. Garcia-Etxarri, A. J. Huber, K. Crozier, J. Aizpurua, and R. Hillenbrand, Nat. Photonics 3, 287 (2009).

${ }^{25}$ E. Cubukcu, E. A. Kort, K. Crozier, and F. Capasso, Appl. Phys. Lett. 89, 093120 (2006).

${ }^{26}$ L. Rogobete, F. Kaminski, M. Agio, and V. Sandoghdar, Opt. Lett. 32, 1623 (2007).

${ }^{27}$ O. L. Muskens, V. Giannini, J. A. Sanchez-Gil, and J. G. Rivas, Nano Lett. 7, 2871 (2007).

${ }^{28}$ N. Engheta, Science 317, 1689 (2007).

${ }^{29}$ N. Engheta, Phys. World 23, 31 (2010).

${ }^{30}$ N. Engheta, A. Salandrino, and A. Alù, Phys. Rev. Lett. 95, 095504 (2005).

${ }^{31}$ Y. Sun, B. Edwards, A. Alù, and N. Engheta, Nat. Mater. 11, 208 (2012).

${ }^{32}$ H. C. van de Hulst, Light Scattering by Small Particles (Dover, New York, 1981).

${ }^{33}$ P. B. Johnson and R. W. Christy, Phys. Rev. B 6, 4370 (1972).

${ }^{34}$ J. D. Bierlein and H. Vanherzeele, J. Opt. Soc. Am. B 6, 622 (1989). 